\title{
Sustained CD28 Expression Delays Multiple Features of Replicative Senescence in Human CD8 T Lymphocytes
}

\author{
Stanley T. Parish $\cdot$ Jennifer E. Wu • Rita B. Effros
}

Received: 16 June 2010 / Accepted: 26 July 2010 / Published online: 19 August 2010

(C) The Author(s) 2010. This article is published with open access at Springerlink.com

\begin{abstract}
CD28 costimulatory signal transduction in $\mathrm{T}$ lymphocytes is essential for optimal telomerase activity, stabilization of cytokine mRNAs, and glucose metabolism. During aging and chronic infection with HIV-1, there are increased proportions of $\mathrm{CD} 8 \mathrm{~T}$ lymphocytes that lack CD28 expression and show additional features of replicative senescence. Moreover, the abundance of these cells correlates with decreased vaccine responsiveness, early mortality in the very old, and accelerated HIV disease progression. Here, we show that sustained expression of CD28, via gene transduction, retards the process of replicative senescence, as evidenced by enhanced telomerase activity, increased overall proliferative potential, and reduced secretion of pro-inflammatory cytokines. Nevertheless, the transduced cultures eventually do reach senescence, which is associated with increased CTLA-4 gene expression and a loss of CD28 cell surface expression. These findings further elucidate the central role of CD28 in the replicative senescence program, and may ultimately lead to novel therapies for diseases associated with replicative senescence.
\end{abstract}

Keywords Human · T lymphocytes · CD28 - CTLA-4 . telomerase $\cdot$ replicative senescence

S. T. Parish · J. E. Wu • R. B. Effros

Department of Pathology and Laboratory Medicine,

David Geffen School of Medicine at UCLA,

10833 Le Conte Avenue,

Los Angeles, CA 90095, USA

R. B. Effros $(\square)$

UCLA AIDS Institute,

David Geffen School of Medicine at UCLA,

10833 Le Conte Avenue,

Los Angeles, CA 90095, USA

e-mail: reffros@mednet.ucla.edu

\section{Introduction}

The CD28 costimulatory receptor, a $44 \mathrm{kDa}$ membrane glycoprotein expressed on nearly all human $\mathrm{T}$ lymphocytes at birth, provides the requisite secondary signal for initiation of an immune response [1]. Binding of CD28 to its CD80 ligands on antigen-presenting cells transduces survival and proliferation signals, as well as induction of interleukin (IL)-2, activation of telomerase, stabilization of mRNA for several cytokines, increased glucose metabolism, and enhanced $\mathrm{T}$ cell migration and homing [2-7]. During aging, there is an accumulation of $\mathrm{T}$ lymphocytes that lack CD28 expression, particularly within the CD8 subset [8]. Increased proportions of $\mathrm{CD} 8+\mathrm{CD} 28-\mathrm{T}$ lymphocytes correlates with multiple negative clinical outcomes, including decreased vaccine responsiveness, reduction in the overall $\mathrm{T}$ cell repertoire, and diminished control over infection [9-11]. Cells with this phenotype have also been observed in younger individuals with HIV/ AIDS, anklylosing spondylitis, and rheumatoid arthritis [12-14]. A variety of studies have confirmed that these CD8+CD28- $\mathrm{T}$ lymphocytes are the in vivo correlates of $\mathrm{CD} 8 \mathrm{~T}$ lymphocytes that have undergone multiple rounds of antigen-driven proliferation in cell culture and have reached the end-stage of replicative senescence [15].

Our previous studies have shown that modification of CD28-related pathways prevented or retarded the process of replicative senescence. For example, constitutive expression the human telomerase catalytic component (hTERT) gene in virus-specific CD8 T lymphocytes led to unlimited proliferation, and enhanced anti-viral effector functions. [16]. Nevertheless, the loss of CD28 expression, although significantly retarded, was not prevented. Prolonged expression of CD28 expression was also documented in studies on long-term cultures of $\mathrm{CD} 8 \mathrm{~T}$ lymphocytes in 
which tumor necrosis factor alpha (TNF- $\alpha$ ) signaling was inhibited. The cultures showed significant increases in telomerase activity, IL-2 gene transcription, and proliferative potential [17]. Furthermore, the secretion of proinflammatory cytokines was significantly decreased, and expression of the p21 cell cycle inhibitor expression was delayed. However, the cultures ultimately did reach replicative senescence, suggesting that manipulation of more upstream events in the senescence pathway, such as CD28 itself, might provide a more robust approach.

CD28 signaling is essential for optimal telomerase activity. Indeed, the decline in telomerase activity as CD8 $\mathrm{T}$ cells progress in culture to replicative senescence precisely parallels the loss of CD28 expression, and blocking CD28 signaling abrogates activation-induced telomerase upregulation [4]. The goal of the current study, therefore, was to investigate the effect of introducing an exogenous CD28 gene into cultures of chronically activated human CD8 T cells. We show that continuous CD28 expression significantly retards the appearance of a majority of the phenotypic and functional changes associated with replicative senescence. The ultimate cessation of proliferation in the transduced cultures, albeit after a significantly prolonged period of active cell division, was associated with an increase in CTLA-4 expression, correlating with a loss of CD28 cell surface expression. These findings further elucidate the central role of CD28 expression changes in the replicative senescence program, and may help in the design of novel therapeutic approaches that target this pathway in clinical situations associated with chronic immune activation.

\section{Materials and Methods}

Cell Cultures Human peripheral blood samples from selfreported healthy donors under 40 years of age were acquired by venipuncture after informed consent, and in accordance with the UCLA IRB. After centrifugation, the layer of peripheral blood mononuclear cells (PBMC) was carefully removed and washed twice in "complete RPMI" (10\% fetal bovine serum, $10 \mathrm{mM}$ Hepes, $2 \mathrm{mM}$ glutamine, $50 \mathrm{IU} / \mathrm{mL}$ penicillin/streptomycin). The EasySep CD8 enrichment kit (Stemcell) was used to isolate CD8+ T cells by negative selection, and purity of the cells was verified by flow cytometry. Cell cultures were established as described previously [18]. Briefly, CD8 T cells were exposed to T cell activation microbeads (anti-CD2/3/28, Miltenyi) with $10 \mu 1$ microbead cocktail added for every $1 \times 10^{6}$ cells, and stimulation repeated every 14 days. Cultures were supplemented with recombinant IL-2 $(20 \mathrm{U} / \mathrm{mL})$. Every 3-4 days, viable cell concentration was determined by trypan blue exclusion, and when the concentration reached $\geq 8 \times 10^{5} / \mathrm{ml}$, cells were subcultivated to a density of $5 \times 10^{5}$ cells $/ \mathrm{ml}$. Population doublings (PD) were determined according to the formula:

$\mathrm{PD}=\log _{2}($ final cell concentration/initial cell concentration $)$

CD28 Gene Transduction The pBABE retroviral supernatants containing either CD28-puromycin, or empty vector-puromycin were provided by the UCLA Vectorcore (Los Angeles, CA). On day 4 following the first stimulation, $1 \times 10^{6} \mathrm{CD} 8 \mathrm{~T}$ cells were isolated and the media was removed by centrifugation. Cells were resuspended in either CD28 or empty vector viral supernatants, supplemented with $8 \mu \mathrm{g} / \mathrm{ml}$ polybrene, and centrifuged at $1,800 \times g$ for $3 \mathrm{~h}$. Cells were incubated in the viral supernatant overnight, at which point they were washed with complete RPMI 1640. A second round of transduction was performed after $24 \mathrm{~h}$ to ensure optimal gene transfer. Transduced cells were selected with pre-titrated concentration of puromycin (Sigma-Aldrich, St. Louis, MO) for 3 days. Transduction efficiency ranged from $5 \%$ to $15 \%$ and gene expression remained stable for the life of the culture.

Flow Cytometry Surface expression of CD4, CD28, CD8, and $\mathrm{CD} 3$ was examined by immunostaining and flow cytometry. Cells were incubated with FITC-conjugated anti-CD4, PE-conjugated anti-CD28, allophycocyanin (APC)-conjugated anti-CD3, and PerCp-conjugated antiCD8 (BD Biosciences, San Jose, CA) at $4^{\circ} \mathrm{C}$ for $20 \mathrm{~min}$, washed, and fixed in PBS containing 1\% paraformaldehyde. Parallel samples were incubated with Ig isotype control antibody or secondary Abs (BD Biosciences). All samples were analyzed on a FACS Calibur flow cytometer (Beckton Dickson). Fluorescence data from at least 25,000 cells were acquired. Analysis of data was performed using Cell Quest Pro (BD Biosciences).

Real-Time Quantitative Polymerase Chain Reaction RNA was isolated using the Qiagen RNAeasy kit (Qiagen, Valencia, CA), and quantified using the Quant-iT Ribogreen RNA Assay Kit (Molecular Probes). cDNAs were synthesized with the iScript cDNA synthesis kit (Bio-Rad) using 500 ng RNA. Real-time quantitative polymerase chain reaction assays were performed using the iQ SYBER Green SuperMix and IQCycler (Bio-Rad). GAPDH was used as an internal control. The sequences were designed with the aid of Beacon Designer software and synthesized at Integrated DNA Technologies, Inc.

CTLA-4 sense-TGAGTTGACCTTCCTAGATGATTCC, anti-sense - CTGGGTTCCGTTGCCTATGC; IL-2 senseTCACCAGGATGCTCACATTTAAGTTTTAC, anti-senseTTCCTCCAGAGGTTTGAGTTCTTCTTC; interferon 
gamma (IFN- $\gamma)$-GAGTGTGGAGACCATCAAGGAA GAC, anti-sense-GCGTTGGACATTCAAGTCAGTT ACC; adenosine deaminase (ADA) sense-CAGGCTAAC TACTCGCTCAACACAG, anti-sense-TGTCCCGTTT GGTCATCTGGTAATC; GAPDH sense-GGTCATGA GTCCTTCCACGATACCA, anti-sense-CCTCAAGATC ATCAGCAATGCCTCCT.

Samples were run in triplicate in a 96-well plate at the following settings: $95^{\circ} \mathrm{C}$ for $15 \mathrm{~s}, 61^{\circ} \mathrm{C}$ for $30 \mathrm{~s}, 72^{\circ} \mathrm{C}$ for $30 \mathrm{~s}$, using single fluorescence measurement.

Cytokine Measurements Culture supernatants were harvested $96 \mathrm{~h}$ post-stimulation and analyzed for IL- 6 and TNF- $\alpha$ in an ELISA Ready-Set-Go (eBioscience, San Diego, CA). All measurements were performed in triplicate wells and in accordance to manufacturer's recommendations.

Telomerase Activity Measurements Telomerase activity was determined using the telomere repeat amplification protocol (TRAP), as previously described in Saldanha et al. [19], with minor modifications.

p21 Quantification $\mathrm{p} 21$ concentration was determined through the use of a p21 quantification assay (Invitrogen, San Diego, CA). All measurements were performed in triplicate wells and in accordance to manufacturer's recommendations.

Statistical Analysis Mean values and standard deviation were calculated for each time-point. Significance was established by using a two-tail Student's $t$ test, and a $p$ value of $<0.05$ is considered significant.

\section{Results}

CD28 Expression Prolongs the Period of Telomerase Activity

CD28 expression provides the necessary secondary signal for multiple immune pathways, including the upregulation of telomerase activity. Previous research on long-term cultures of CD8 $\mathrm{T}$ cells has demonstrated that by the third and all subsequent stimulations telomerase activity was undetectable, and the progressive reduction with each stimulation parallels the decline in CD28 expression [4]. Moreover, preincubation of the APC with antibodies to the B7 ligands abolishes telomerase activity, further confirming the importance of CD28 signal transduction. Since gene transduction with hTERT, which prevented replicative senescence, was nevertheless not able to ultimately prevent loss of CD28 [16], we tested the converse situation. Indeed, our data show that
CD28 gene transduction was associated with the maintenance of telomerase activity to at least the 7 th round of stimulation. The telomerase activity of the cultures was measured 4 days after the 7th stimulation, at which time the empty vector control cultures, contained $<10 \% \mathrm{CD} 28+$ $\mathrm{T}$ cells (Fig. 1). Sustained CD28 expression clearly resulted in significantly higher levels of telomerase activity even after multiple rounds of activation, as compared with the minimal telomerase activity in the control cultures. This was evident in pairs of cultures tested from all four donors, with the mean fold increase over all donor cultures being highly significant (Table 1). These data are consistent with our previous study showing that the delayed loss of constitutive CD28 expression, by inhibiting TNF- $\alpha$, also led to prolonged telomerase activity [17].

CD28 Gene Transduction Prevents Senescence-Associated Functional and Phenotypic Changes

In addition to its importance in telomerase upregulation, CD28 is essential for stabilization of several cytokine mRNAs [2]. Cultures of senescent CD8 T cells have been documented to undergo multiple changes in cytokine secretion patterns, most notably significant increases in TNF- $\alpha$ and IL-6 [20], and concomitant decreases in IFN- $\gamma$ and IL-2 production [21]. IL-2, in particular, is closely linked to $\mathrm{CD} 28$, as the expression of $\mathrm{CD} 28$ has been shown to be necessary for IL-2 signal transduction [22, 23]. Moreover, a report on the short term outcome of introducing exogenous CD28 into cultures of CD28ngative cells showed rescue of IL-2 production over 3 weeks in cell culture [24]. Given the effect of sustained CD28 expression on telomerase activity, we hypothesized that this strategy might also prevent the senescenceassociated changes in cytokine production. We compared the concentration of both TNF- $\alpha$ and IL- 6 in the super-

Fig. 1 Sustained CD28 expression is associated with increased telomerase activity. On day 4 after the 7 th stimulation, lysates were prepared from CD28and empty vector-transduced cultures. Telomerase activity for 2,500 cell-equivalents was analyzed using the TRAP assay, as described in "Materials and Methods". Image of the TRAP gel from a representative experiment is shown

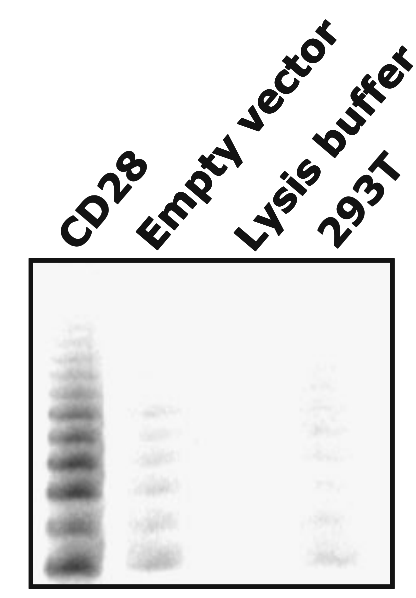


Table I Sustained CD28 expression maintains telomerase activity

\begin{tabular}{ll}
\hline Donor & $\begin{array}{l}\text { Fold increase } \\
\text { CD28/Empty vector }\end{array}$ \\
\hline 1 & 2.34 \\
2 & 1.92 \\
3 & 2.45 \\
4 & 1.81 \\
Mean $\pm \mathrm{SD}$ & $2.13 \pm 0.311$ \\
$p$ value & 0.00034 \\
\hline
\end{tabular}

Telomerase activity for each donor's culture was determined as the fold increase over its respective control (empty vector) at 2,500 cell equivalents, 4 days after 7 th stimulation. The mean fold increase is given and $p$ value determined compared with control

natants 14 days after stimulation for CD28-transduced ( $n=$ $5)$ and vector-transduced $(n=5)$ cultures at $\sim 18 \mathrm{PD}$, the time-point at which the control cultures reached senescence. Whereas TNF- $\alpha$ and IL-6 protein levels were high late in empty vector culture (17 PD), consistent with our previous findings [20], the CD28-transduced cultures showed drastically lower concentrations in both cytokines (Fig. 2a, b). Conversely, the levels of IFN- $\gamma$ and IL-2 message were significantly higher in CD28-transduced cultures as compared with the senescent empty vector cultures (Fig. 2c, d). We also investigated the effect of sustained CD28 expression on ADA, which, like CD28 is a critical component of the immunological synapse [25]. In our previous studies, we showed that both ADA surface expression and activity were significantly greater in CD8+CD28+ (vs. CD8+CD28-) T cells, and were lost as CD8 T lymphocytes approached senescence [26]. We now extend those observations by comparing CD28transduced cultures $(n=5)$ and vector control cultures $(n=5)$ at $\sim 18 \mathrm{PD}$, for ADA gene expression. Figure $2 \mathrm{e}$ shows that sustained expression of CD28 is associated with significantly enhanced ADA expression. Thus, CD28 gene transduction results in the reversal of multiple changes associated with replicative senescence.

\section{Continuous CD28 Expression Increases the Proliferative Potential of CD8 T Cells}

With chronic exposure to antigen, human CD8 $\mathrm{T}$ cells undergo a finite number of population doublings, ultimately reaching the irreversible cell cycle arrest known as replicative senescence [13]. The signature genetic change associated with this end-stage of $\mathrm{T}$ cell differentiation is the permanent loss of CD28 expression. Indeed, CD8+CD28$\mathrm{T}$ cells, whether arising in vitro or in vivo, have shortened telomeres, no telomerase activity, and are unable to proliferate $[12,27]$. We therefore hypothesized that sustained CD28 expression would extend the proliferative potential of CD8 $\mathrm{T}$ cells. Indeed, our data show that there was a significant increase $(P<0.001)$ in the proliferative potential in the CD28-transduced cultures (Fig. 3a). In fact, we observed an average $2^{20}$-fold greater expansion of the cells in the cultures that had been transduced with CD28, and this number is probably an underestimate, since we abstained from performing cell counts during the period of drug selection. We noted, however, that following this extensive expansion, CD28 surface expression precipitously declined, and the cells stopped dividing. Interestingly, in contrast to unmanipulated $\mathrm{T}$ lymphocyte cultures, where both CD28 protein and gene expression are irreversibly suppressed at senescence, the CD28-transduced cultures that ceased dividing lost only the surface CD28 expression, with no change in gene expression (data not shown). The possibility that the ultimate growth arrest of the CD28-transduced cultures was irreversible was verified by the increased expression of the senescence-associated cell cycle inhibitor, p21, late in culture (Fig. 3b).

CTLA-4 Increases with Culture Age Correlates with CD28 Cell Surface Expressional Loss

To explore the underlying mechanism for the loss of CD28 surface expression in the transduced cultures, we investigated the possible role CTLA-4, which is known to compete with the CD28 ligands on APC, and is involved with the down-regulation of immune responses after $\mathrm{T}$ cell activation [28]. Interestingly, elderly persons show increased proportions of $\mathrm{CD} 8+\mathrm{CD} 28-$ regulatory $\mathrm{T}$ cells that express CTLA-4 [29]. Furthermore, persons with HIV/AIDS, which is considered a model of premature immunological aging [30], have increased proportions of CD4+CD28- T cells that express CTLA-4 [31, 32]. To determine the possible role of CTLA-4 in the CD28related changes associated with replicative senescence, we first evaluated the level of CTLA-4 message in early and late unmanipulated cultures, which lose CD28 protein and gene expression with increasing population doublings. In these cultures, we observed that CTLA-4 transcripts, as measured by real-time PCR, are significantly increased with culture age (Fig. 4). A distinctly different scenario occurred in the CD28-transduced cultures that eventually ceased dividing after an extensive period of expansion. Indeed, although there was an increase in CTLA-4 gene expression at the time-point when CD28 surface expression was lost, this changes was not associated with any reduction in the message levels of the exogenously introduced gene (data not shown). Thus, the nonproliferative state that occurred in the transduced cultures may be under different regulatory control mechanisms as compared with unmanipulated CD8 T cell cultures that reach replicative senescence. 

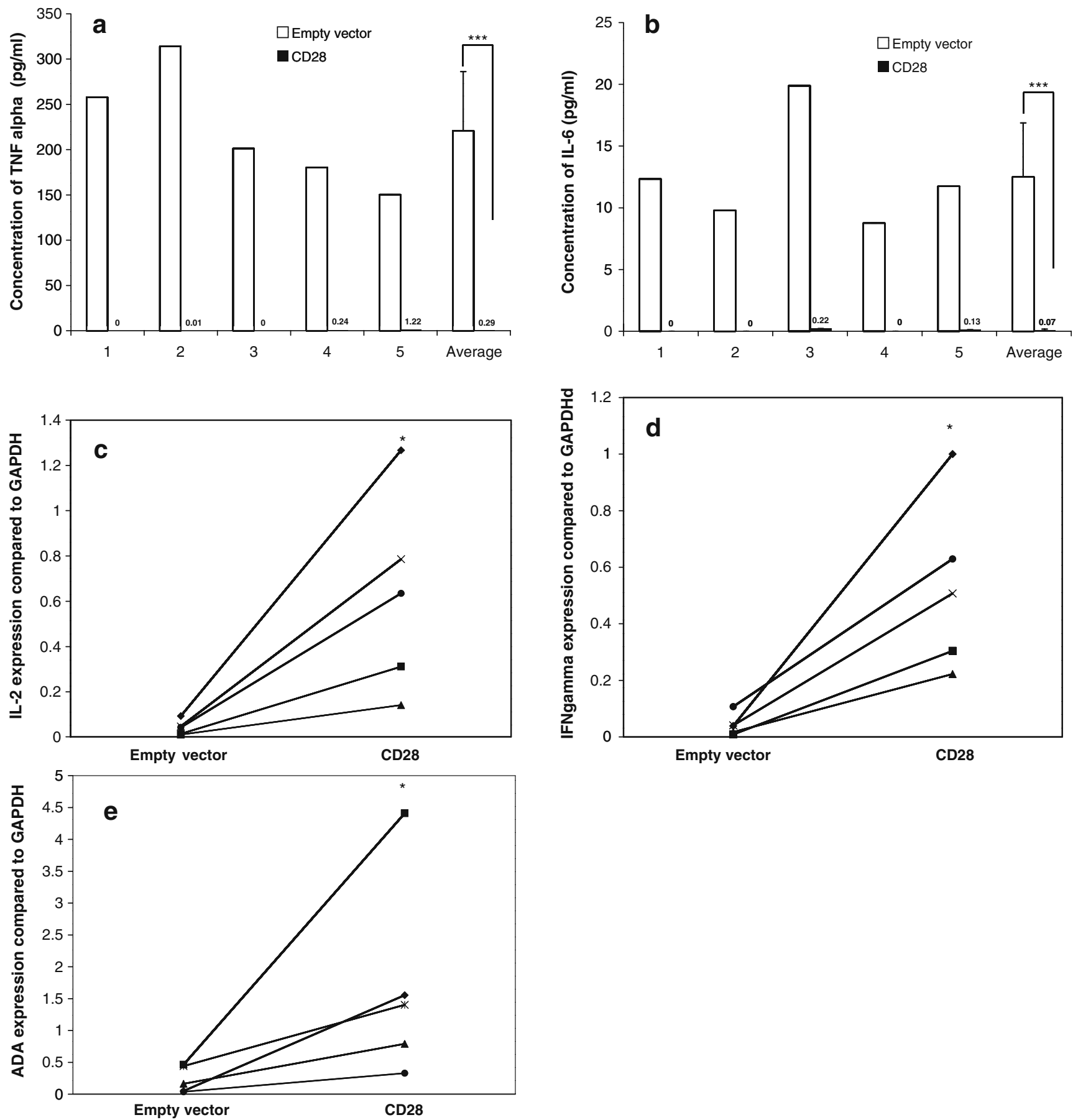

Fig. 2 Effect of sustained CD28 expression on senescence-associated cytokine patterns and ADA expression. Cytokine profiles were evaluated for CD28-transduced $(n=5)$ and vector-transduced $(n=5)$ cultures at $\sim 18 \mathrm{PD}$, the time-point at which the control culture reached senescence. a and $\mathbf{b}$ IL-2 and IFN- $\gamma$ mRNA levels determined by real-

time quantitative PCR; results expressed relative to GAPDH; $\mathbf{c}$ and $\mathbf{d}$ concentrations of IL- 6 and TNF- $\alpha$ measured by ELISA. e ADA mRNA levels determined by real-time quantitative PCR. ${ }^{*} P<0.05$; $* * * p<0.001$

\section{Discussion}

The current study provides the first documentation that introduction of an exogenous CD28 gene into human CD8 $\mathrm{T}$ lymphocytes delays the onset of replicative senescence

and the associated genetic and functional changes normally observed in cell culture. Importantly, telomerase activity, which is central to the telomere-loss that signals replicative senescence, is maintained through at least the 7 th round of stimulation, in contrast to the previously documented loss 


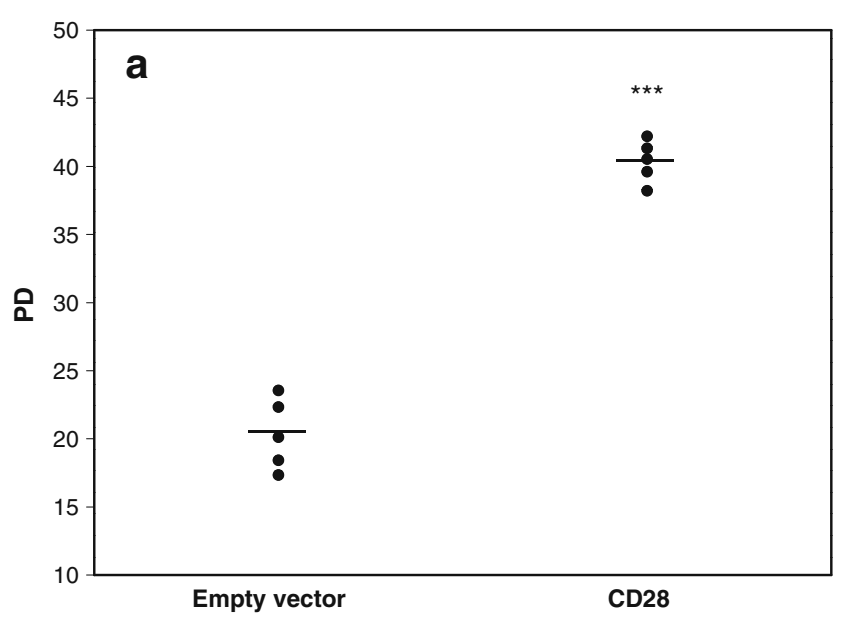

Fig. 3 Sustained CD28 increases the proliferative potential of CD8 T lymphocytes. Long-term CD8 T lymphocyte cultures were established from PBMC of healthy donors $(n=5)$, as described in the "Materials and Methods". After 4 days, CD8 T cells were transduced with either empty vector or vector containing the $\mathrm{CD} 28$ transgene, and population

by the 3rd stimulation [4]. Our data also provide new insights into the role of CTLA-4 in the senescence program. In the absence of gene transduction, CTLA-4 message increases with culture age, and in those cultures, both protein and message of CD28 progressively declines. By contrast, when the proliferative activity in the CD28transduced cultures eventually declines, the increased CTLA-4 expression is not associated with altered CD28 gene transcription, but rather, correlates only with a loss of CD28 cell surface expression. These studies, therefore, suggest that there is a post-translation down-regulation of CD28 that remains to be elucidated. Further investigation

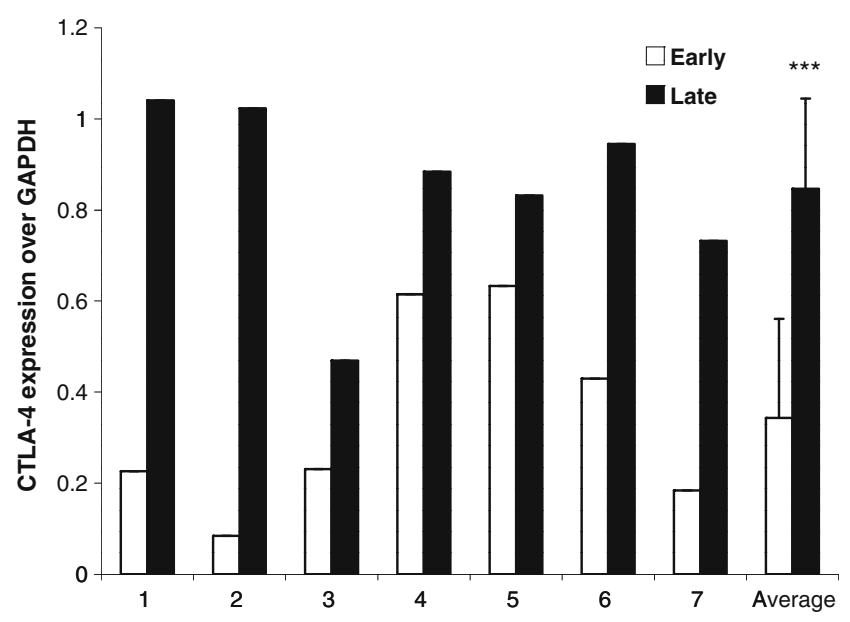

Fig. 4 CTLA-4 expression increases with culture age. Long-term CD8 T lymphocyte cultures were established as described in "Materials and Methods". On day 4 after stimulation cell pellets from early (i.e., $10 \%$ of in vitro lifespan) and late (i.e., $90 \%$ of in vitro lifespan) were collected for real-time quantitative PCR, in which CTLA-4 expression was compared with that of GAPDH. $* * * P<0.001$

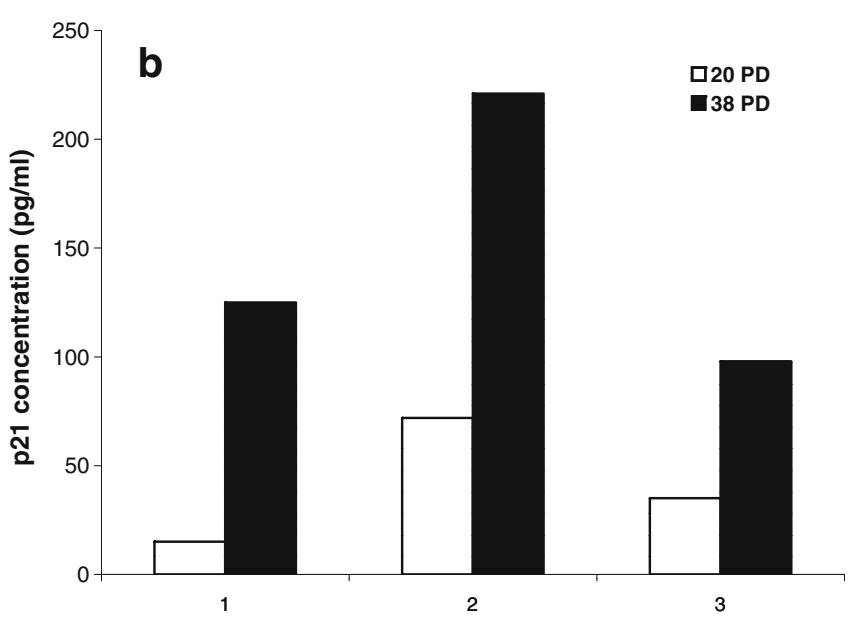

doublings $(P D)$ followed until replicative senesce was reached. a Population doublings for both types of cultures are shown, with the mean PD indicated by the horizontal line, $* * * P<0.00$. b p 21 concentration in three representative CD28-transduced cultures at 20 and $38 \mathrm{PD}$

into $\mathrm{CD} 28$ regulation could provide new insights into the population of $\mathrm{CD} 8+\mathrm{CD} 28-\mathrm{T}$ cells present in vivo that are associated with a variety of deleterious health effects in humans, including reduced influenza vaccine responses, various forms of cancer [9, 33-35], and more rapid progression to AIDS [36].

We observed that sustained CD28 expression, via gene transduction, leads to the long-term maintenance of telomerase activity. Previous research has shown that CD28 is essential for optimal telomerase activity, and that, not only do CD8 T cells lose telomerase in parallel with the loss of CD28 expression, but also inhibition of the B7 ligands on APC significantly reduces telomerase activity [4]. Enhanced telomerase activity has been shown to be essential for the immune control of HIV both in vitro and in vivo [16]. Here, we show that that telomerase activity of chronically stimulated CD8 T cells can be maintained by the continuous expression of exogenous CD28 (Fig 1). The present study is consistent with our previous reports showing that sustained telomerase activity, by either gene transduction or exposure to a chemical telomerase activator, is associated with significantly enhanced proliferative potential $[16,37]$.

In addition to telomerase activity, CD28 signaling is essential for production of a variety of cytokines. As T cells approach replicative senescence in culture, not only is CD28 expression lost, but there is a dramatic alteration in the profile of various cytokines. Specifically, as CD8 T cells approach senescence, they secrete increasing amounts of both the pro-inflammatory cytokines, TNF- $\alpha$ and IL-6. We observed that sustained $\mathrm{CD} 28$ expression greatly reduces the secretion of these two cytokines, while increasing expression of IFN- $\gamma$ and IL-2 (Fig. 2). These results extend 
our previous studies documenting reduced IL-6 secretion when CD28 loss was delayed via TNF- $\alpha$ inhibition [17]. In that study, it was not possible to differentiate whether the reduction in IL-6 was due to the direct effect of higher CD28 expression or to an indirect effect of TNF- $\alpha$ inhibition. By contrast, the current study shows clearly show that CD28 is directly involved in the maintenance of a "more youthful" cytokine secretion pattern.

Our studies also yield additional insights into the potential role of CTLA-4 in the generation of senescent CD8 T lymphocytes. Indeed, the data in Fig. 4 provide the first evidence of an increase in CTLA-4 expression in human CD8 T lymphocytes as they approach replicative senescence, suggesting yet another mechanism by which CD28 is irreversibly down regulated after chronic activation in vitro. We have previously documented the potential involvement of TNF- $\alpha$ and adenosine in this downregulation; combining those observations with the current data, we conclude that multiple pathways may contribute to the generation of $\mathrm{CD} 8+\mathrm{CD} 28-\mathrm{T}$ lymphocytes in vivo. Our current demonstration of three concomitant changes-loss of exogenous CD28 surface expression, upregulation of CTLA-4 message, and the sudden cessation of proliferationunderscores the importance of the surface expression of CD28 protein in delivering proliferation signals. Indeed, despite the sustained gene expression of $\mathrm{CD} 28$, the cells were unable to enter cell cycle, as verified by an increase in p 21 concentration. While, CD56 expression has been associated with senescence [38], we and others have shown that cultures of CD8 $\mathrm{T}$ cells that reach replicative sensescence lack CD28 and accumulate p21 and p16 [16, 39]. Future studies will focus on analysis of the downstream signaling mechanism associated with the increase in CTLA-4, and its ultimate repression of $\mathrm{CD} 28$ protein expression. In our cultures, we have not been able to detect CTLA- 4 on the cell surface or intracellularly, possibly reflecting its rapid turnover or degradation.

In summary, our results further elucidate the central role of CD28 signaling in chronically activated human CD8 T lymphocytes. As these cells progress to replicative senescence in culture, CD28 expression is lost, and this loss is associated with a variety of phenotypic and functional changes. Our data show that sustained CD28 expression, via gene transduction, significantly retards many of these changes. Based on the growth arrest that was ultimately observed even in the transduced cultures, we conclude that CTLA-4 provides another possible mechanism that contributes to the irreversible loss of CD28 associated with senescence. Future studies that test the effects of CTLA-4 inhibition in combination with $\mathrm{CD} 28$ gene transduction should provide additional insights into the complex regulatory influences on CD8 T cell senescence. Ultimately, these findings may, therefore, lead to novel therapeutic approaches to prevent the production of senescent CD8 T cells in vivo due to aging, cancer, and HIV/AIDS.

Acknowledgements This research was supported by the following NIH grants: AG023720 and AI060362 (RBE).

Open Access This article is distributed under the terms of the Creative Commons Attribution Noncommercial License which permits any noncommercial use, distribution, and reproduction in any medium, provided the original author(s) and source are credited.

\section{References}

1. Lenschow DJ, Walunas TL, Bluestone JA. CD28/B7 system of T cell costimulation. Annu Rev Immunol. 1996;14:233-58.

2. Lindstein $T$, et al. Regulation of lymphokine messenger RNA stability by a surface-mediated T cell activation pathway. Science. 1989;244(4902):339-43.

3. Powell JD, et al. Molecular regulation of interleukin-2 expression by CD28 co-stimulation and anergy. Immunol Rev. 1998; 165:287-300.

4. Valenzuela HF, Effros RB. Divergent telomerase and CD28 expression patterns in human CD4 and CD8 T cells following repeated encounters with the same antigenic stimulus. Clin Immunol. 2002;105(2):117-25.

5. Frauwirth KA, et al. The CD28 signaling pathway regulates glucose metabolism. Immunity. 2002;16(6):769-77.

6. June $\mathrm{CH}$, et al. The $\mathrm{B} 7$ and $\mathrm{CD} 28$ receptor families. Immunol Today. 1994;15(7):321-31.

7. Shimizu $Y$, et al. Crosslinking of the $\mathrm{T}$ cell-specific accessory molecules CD7 and CD28 modulates T cell adhesion. J Exp Med. 1992;175(2):577-82.

8. Posnett DN, et al. Clonal populations of T cells in normal elderly humans: the T cell equivalent to "benign monoclonal gammapathy". J Exp Med. 1994;179(2):609-18.

9. Goronzy JJ, et al. Value of immunological markers in predicting responsiveness to influenza vaccination in elderly individuals. J Virol. 2001;75(24):12182-7.

10. Hadrup SR, et al. Longitudinal studies of clonally expanded CD8 $\mathrm{T}$ cells reveal a repertoire shrinkage predicting mortality and an increased number of dysfunctional cytomegalovirus-specific $\mathrm{T}$ cells in the very elderly. J Immunol. 2006;176(4):2645-53.

11. Saurwein-Teissl M, et al. Lack of antibody production following immunization in old age: association with $\mathrm{CD} 8(+) \mathrm{CD} 28(-) \mathrm{T}$ cell clonal expansions and an imbalance in the production of Th1 and Th2 cytokines. J Immunol. 2002;168(11):5893-9.

12. Effros RB, et al. Shortened telomeres in the expanded CD28-CD8 + cell subset in HIV disease implicate replicative senescence in HIV pathogenesis. AIDS. 1996;10(8):F17-22.

13. Effros RB. From Hayflick to Walford: the role of T cell replicative senescence in human aging. Exp Gerontol. 2004;39(6):885-90.

14. Schmidt D, Goronzy JJ, Weyand CM. CD4+ CD7- CD28- T cells are expanded in rheumatoid arthritis and are characterized by autoreactivity. J Clin Invest. 1996;97(9):2027-37.

15. Effros RB. Replicative senescence of CD8 T cells: effect on human ageing. Exp Gerontol. 2004;39(4):517-24.

16. Dagarag M, et al. Genetic manipulation of telomerase in HIVspecific CD8+ T cells: enhanced antiviral functions accompany the increased proliferative potential and telomere length stabilization. J Immunol. 2004;173(10):6303-11.

17. Parish ST, Wu JE, Effros RB. Modulation of $\mathrm{T}$ lymphocyte replicative senescence via TNF-\{alpha\} inhibition: role of caspase-3. J Immunol. 2009;182(7):4237-43. 
18. Perillo NL, et al. Human T lymphocytes possess a limited in vitro life span. Exp Gerontol. 1989;24(3):177-87.

19. Saldanha SN, Andrews LG, Tollefsbol TO. Analysis of telomerase activity and detection of its catalytic subunit, hTERT. Anal Biochem. 2003;315(1):1-21.

20. Effros RB, et al. The role of CD8+ T-cell replicative senescence in human aging. Immunol Rev. 2005;205:147-57.

21. Rink L, Cakman I, Kirchner H. Altered cytokine production in the elderly. Mech Ageing Dev. 1998;102(2-3):199-209.

22. Fraser JD, et al. Regulation of interleukin-2 gene enhancer activity by the $\mathrm{T}$ cell accessory molecule CD28. Science. 1991;251 (4991):313-16.

23. Verweij CL, Geerts M, Aarden LA. Activation of interleukin-2 gene transcription via the T-cell surface molecule CD28 is mediated through an NF-kB-like response element. J Biol Chem. 1991;266(22):14179-82.

24. Topp MS, et al. Restoration of CD28 expression in CD28- CD8+ memory effector $\mathrm{T}$ cells reconstitutes antigen-induced IL-2 production. J Exp Med. 2003;198(6):947-55.

25. Pacheco R, et al. CD26, adenosine deaminase, and adenosine receptors mediate costimulatory signals in the immunological synapse. Proc Natl Acad Sci USA. 2005;102(27):9583-8.

26. Parish ST, et al. Adenosine deaminase modulation of telomerase activity and replicative senescence in human CD8 T lymphocytes. J Immunol. 2010;184(6):2847-54.

27. Vaziri H, et al. Loss of telomeric DNA during aging of normal and trisomy 21 human lymphocytes. Am J Hum Genet. 1993;52(4):661-7.

28. Rudd CE, Taylor A, Schneider H. CD28 and CTLA-4 coreceptor expression and signal transduction. Immunol Rev. 2009;229(1):12-26.
29. Simone R, Zicca A, Saverino D. The frequency of regulatory CD3 $+\mathrm{CD} 8+\mathrm{CD} 28-\mathrm{CD} 25+\mathrm{T}$ lymphocytes in human peripheral blood increases with age. J Leukoc Biol. 2008;84(6):1454-61.

30. Appay V, et al. Accelerated immune senescence and HIV-1 infection. Exp Gerontol. 2007;42(5):432-7.

31. Stone SF, Price P, French MA. Dysregulation of CD28 and CTLA-4 expression by $\mathrm{CD} 4 \mathrm{~T}$ cells from previously immunodeficient HIV-infected patients with sustained virological responses to highly active antiretroviral therapy. HIV Med. 2005;6(4):278-83.

32. Steiner K, et al. Enhanced expression of CTLA-4 (CD152) on CD4+ T cells in HIV infection. Clin Exp Immunol. 1999;115(3):451-7.

33. Sze DM, et al. Clonal cytotoxic T cells are expanded in myeloma and reside in the CD8(+)CD57(+)CD28(-) compartment. Blood. 2001;98(9):2817-27.

34. Tsukishiro T, Donnenberg AD, Whiteside TL. Rapid turnover of the CD8(+)CD28(-) T-cell subset of effector cells in the circulation of patients with head and neck cancer. Cancer Immunol Immunother. 2003;52(10):599-607.

35. Hakansson A, et al. Biochemotherapy of metastatic malignant melanoma. On down-regulation of CD28. Cancer Immunol Immunother. 2002;51(9):499-504.

36. Cao W, et al. Premature aging of $\mathrm{T}$ cells is associated with faster HIV-1 disease progression. J Acquir Immune Defic Syndr. 2009;50(2):137-47.

37. Fauce SR, et al. Telomerase-based pharmacologic enhancement of antiviral function of human CD8+ T lymphocytes. J Immunol. 2008;181(10):7400-6.

38. Lemster BH, et al. Induction of CD56 and TCR-independent activation of T cells with aging. J Immunol. 2008;180(3):1979-90.

39. Liu Y, et al. Expression of p16(INK4a) in peripheral blood T-cells is a biomarker of human aging. Aging Cell. 2009;8(4):439-48. 